\title{
Photodynamic Antimicrobial Chemotherapy (PACT) Using Phenothiazine Derivatives as Photosensitizers Against Leishmania braziliensis
}

\author{
Artur F. Barbosa, Msc, ${ }^{1,2,3,4}$ Bruno B. Sangiorgi, Biol, ${ }^{2}$ Suely L. Galdino, PhD, ${ }^{3}$ Manoel Barral-Netto, MD, PhD, ${ }^{2}$ \\ Ivan R. Pitta, PhD, ${ }^{3}$ and Antônio L. Pinheiro, PhD $^{1,5^{*}}$ \\ ${ }^{1}$ Center of Biophotonics, School of Dentistry, Federal University of Bahia (UFBA), 62 Araujo Pinho Ave, Canela, \\ Salvador, BA 40110-150, Brazil \\ ${ }^{2}$ Centro de Pesquisas Goncalo Moniz (FIOCRUZ), Rua Waldemar Falcão 121, Candeal, Salvador, BA 40296-710, \\ Brazil \\ ${ }^{3}$ Laboratory Planning and Synthesis of Drugs, Federal University of Pernambuco, 1235 Prof. Moraes Rego Ave, Cidade \\ Universitária, Recife, PE 50670-901, Brazil \\ ${ }^{4}$ São Camilo School, 102 Visconde de Itaborai Street, Amaralina, Salvador, BA 41900-000, Brazil \\ ${ }^{5}$ National Institute of Optics and Photonics, University of São Paulo, 400 Trabalhador Sancarlense Ave, São Carlos, SP \\ 13560-970, Brazil
}

\begin{abstract}
Background and Objective: Cutaneous and mucocutaneous leishmaniasis are diseases characterized by skin or mucosal manifestations. In the new world, Leishmania braziliensis is the main etiological agent of cutaneous leishmaniasis, condition that may evolve to the mucocutaneous form. The therapeutic arsenal routinely employed to treat infected patients is unsatisfactory, especially for pentavalent antimonials, treatment recommended by the WHO, as they are often highly toxic, poorly tolerated and of variable effectiveness. This work aimed to evaluate in vitro the effectiveness of photodynamic antimicrobial chemotherapy as a new approach for the treatment of leishmaniasis.
\end{abstract}

Materials and Methods: A laser $(\lambda=660 \mathrm{~nm}, 40 \mathrm{~mW}$, $4.2 \mathrm{~J} / \mathrm{cm}^{2}$, and $8.4 \mathrm{~J} / \mathrm{cm}^{2}$, CW) associated to phenothiazine's derivatives ( 5 and $10 \mu \mathrm{g} / \mathrm{ml}$, toluidine blue $\mathrm{O}$, methylene blue, or phenothiazine) on the promastigote forms of $L$. braziliensis in a single session. Samples were removed and analyzed in a hemocytometer 72 hours after PACT and viability of the parasites was assessed in quadruplicates.

Results: An important decrease in the number of viable parasites on all treated groups in comparison to their controls was observed as all tested compounds lead to significant parasite lethality being the highest lethality achieved with $10 \mu \mathrm{g} / \mathrm{ml}$ of TBO. No lethality was observed on groups treated with laser or with any of the compounds separately.

Conclusions: TBO presented higher parasite lethality in comparison to $\mathrm{MB}$ with impressive reduction from 1 hour to 5 minutes of pre-incubation time. Lasers Surg. Med. 44:850-855, 2012. (C) 2012 Wiley Periodicals, Inc.

Key words: laser-therapy; leishmaniasis; methylene blue; toluidine blue $\mathrm{O}$; treatment

\section{INTRODUCTION}

Leishmaniasis are protozoan parasitic infections transmitted to mammals, including humans, by phlebotomine sandflies and clinically classified in three forms: visceral, cutaneous, and mucocutaneous [1]. Leishmaniasis threatens about 350 million people in 88 countries worldwide and 12 million people are believed to be currently infected, with about 1-2 million estimated new cases occurring every year [2].

Cutaneous leishmaniasis is mainly caused by Leishmania major in the old world and by Leishmania amazonensis or Leishmania braziliensis in the Americas, in particular in Brazil [2-4]. L. braziliensis is the etiologic agent of American cutaneous leishmaniasis (ACL), which is characterized by its chronicity and the possibility to metastasize leading to the mucocutaneous clinical form [5].

Current treatment for leishmaniasis relies mainly on chemotherapy, as no efficient vaccine is available. Pentavalent antimonials meglumine antimoniate and sodium stibogluconate have been considered the first option of treatment of leishmaniasis since the 1940s [1,6]. Amphotericin B is the second drug of choice when there is no response to treatment with antimonials or when it is not possible to use it. The efficacy of pentamidine, the third drug of choice, is less well known. Pentavalent

Conflict of Interest Disclosures: All authors have completed and submitted the ICMJE Form for Disclosure of Potential Conflicts of Interest and none were reported.

*Corresponding to: Antônio Luiz B. Pinheiro, PhD, Center of Biophotonics, School of Dentistry, Federal University of Bahia, 62 Araujo Pinho Ave, Canela, Salvador, BA 40110-150, Brazil.

E-mail: albp@ufba.br

Accepted 4 November 2012

Published online 26 November 2012 in Wiley Online Library

(wileyonlinelibrary.com).

DOI 10.1002/lsm.22099 
antimonials are expensive, toxic many organs. Furthermore, the administration of antimonials is forbidden in many situations such as during both pregnancy and lactation, in very small children, in case of hypersensitivity to the drugs, and in people suffering from certain chronic diseases $[7,8]$. Several side effects from use of these compounds may be found elsewhere in the literature [2].

Recently, photodynamic antimicrobial chemotherapy (PACT) has been studied for the treatment of leishmaniasis [9-11]. PACT is a potentially applicable, safe, and affordable option for treating patients suffering from many conditions being an attractive option to the use of conventional antimicrobial chemotherapy [12]. PACT neither induces resistant strains nor demands multiple sessions of treatment [13,14].

Phenothiazines, nitrogen-, and sulfur-containing tricyclic compounds, are known for over a 100 years. Up to now over 5,000 phenothiazine's derivatives have been obtained and this class of organic compounds became extremely important due to their varied biological and chemical properties. Recent reports dealt with promising anticancer, antibacterial, antiplasmid, multidrug resistance (MDR) reversal activities, and potential treatment in Alzheimer's and Creutzfeldt-Jakob diseases of classical phenothiazines [15]. Phenothiazines are relatively inexpensive, widely available, well-tolerated, and non-toxic compounds. New phenothiazines exhibit promising antibacterial, antifungal, anticancer, antiviral, anti-inflammatory, antimalarial, antifilarial, trypanocidal, anticonvulsant, analgesic, immunosuppressive, and multidrug resistance reversal properties [15].

The present investigation aimed to evaluate the effectiveness of PACT against the promastigote form of L. braziliensis, as a new approach to therapy against human cutaneous leishmaniasis.

\section{MATERIALS AND METHODS}

\section{Chemicals}

$\mathrm{MB}$ and TBO were purchased from Sigma-Aldrich Corporation (Milwaukee, WI). Three compounds were used: MB, TBO, and a 1:1 mixture of $\mathrm{MB}+\mathrm{TBO}$, here named as phenothiazine-MB-TBO. The stock solutions were diluted in distilled water (10 or $20 \mu \mathrm{g} / \mathrm{ml})$.

\section{Culture Promastigote Forms}

L. braziliensis promastigotes (strain MHOM/BR/01/ BA788 - cultivated and maintained by the Integrated Laboratory of Microbiology and immunoregulation) were cultivated in Schneider's Insect Medium (Sigma Chemical Co., St. Louis, MO) supplemented with $20 \%$ heat-inactivated fetal bovine serum (Gibco, Carlsbad, CA), L-glutamine $(2 \mathrm{mM})$, penicillin $(100 \mathrm{U} / \mathrm{ml})$, streptomycin $(100 \mu \mathrm{g} / \mathrm{ml})$ at $26^{\circ} \mathrm{C}$ (Thermostat cabinets-LS Scientific, Ikeja, Lagos, Nigeria) for 5-7 days when the parasites reached the stationary-phase. After reaching the stationary-phase, the culture was sedimented by centrifugation (5810 R, Eppendorf, Hamburg, Germany, 10 minutes, $4^{\circ} \mathrm{C}$, 4,000 rpm), suspended in $1 \mathrm{ml}$ of the same medium and quantified in a Neubauer chamber (LO-Laboroptik GmbH, Friedrichsdorf,
Germany) with the aid of a microscope (Nikon Eclipse E200, Melville, NY). Enough medium was added in order to adjust the parasitic concentration to $2 \times 10^{6}$ promastigotes/ $\mathrm{ml}[4]$.

\section{Leishmanicidal Activity Against Promastigotes Forms}

The determination of the leishmanicidal activity was performed in 96-well plates (Tesplate 96F-TPP, Zollstrasse, Trasadingen, Switzerland). The compounds were tested in quadruplicate in two concentrations 5 or $10 \mu \mathrm{g} / \mathrm{ml}$. The parasitic concentration in the wells was that of $2 \times 10^{6}$ promastigotes $/ \mathrm{ml}$. A negative control was performed with four wells containing only parasites and the incubation medium. After 72 hours of incubation at $26^{\circ} \mathrm{C}$, $10 \mu \mathrm{l}$ of each well was diluted in $90 \mu \mathrm{l}$ of the PBS and the parasites were quantified in a Neubauer chamber. The data obtained from this quantification were plotted in a graph using Microsoft Excel and the values of the lethality of the treated groups were obtained through the difference in percentage of viable parasites in the groups treated with the amount of viable parasites in the control group.

\section{Photodynamic Antimicrobial Chemotherapy (PACT)}

Metacyclic promastigotes were pre-incubated during either 5 or 60 minutes, in darkness, with the different concentrations of the photosensitizers in Schneider's Drosophila medium ( $\mathrm{pH}$ 7.2) with $10 \%$ FBS in 96-well plates. After pre-incubation each well was irradiated with a diode laser which has a semiconductor active medium InGaAlP $\left(\lambda=660 \mathrm{~nm}, 40 \mathrm{~mW}, 4.2 \mathrm{~J} / \mathrm{cm}^{2}, \mathrm{CW}\right.$, TwinFlex ${ }^{\odot}$, MMOptics, São Carlos, São Paulo, Brazil). After 72 hours of incubation at $26^{\circ} \mathrm{C}, 10 \mu \mathrm{l}$ of each well was diluted in $90 \mu \mathrm{l}$ of the formaline $10 \%$ and the parasites were quantified in a Neubauer chamber. The data obtained from this quantification were plotted in a graph using Microsoft Excel and the values of the lethality of the treated groups were obtained through the difference in percentage of viable parasites in the groups treated with the amount of viable parasites in the control group. Four protocols were carried out: I, pre-incubation of 5 and 2 minutes of irradiation $\left(4.2 \mathrm{~J} / \mathrm{cm}^{2}\right)$; II, pre-incubation of 5 and 4 minutes of irradiation $\left(8.4 \mathrm{~J} / \mathrm{cm}^{2}\right)$; III, pre-incubation of 60 and 2 minutes of irradiation $\left(4.2 \mathrm{~J} / \mathrm{cm}^{2}\right)$; and IV, pre-incubation of 60 and 4 minutes of irradiation $\left(8.4 \mathrm{~J} / \mathrm{cm}^{2}\right)$.

\section{Crystal Violet Toxicity Assay}

In order to assess the toxicity of certain phenothiazines concentrations to human cells, we made a crystal violet toxicity assay from macrophages derived from peripheral blood mononuclear cells (PBMC). PBMC were obtained from blood bags processed with buffy coat, donated from the local hemocenter (Fundação de Hematologia e Hemoterapia da Bahia), by a gradient centrifugation on FicollHypaque (GE Healthcare, Uppsala, Sweden) at 1,500 rpm with room temperature. The PBMC collected were then washed with PBS following centrifugations at 1,400, 1,300, and 1,200 rpm. For macrophage differentiation, 
the PBMC remaining were disposed in a 96-well dish with $200 \mu \mathrm{l}$ of RPMI (LGCBIO, Cotia, SP, Brazil) with $10 \%$ inactivated $\mathrm{AB}$ human serum (Sigma Chemical Co.), at concentration of $10^{5} \mathrm{PBMC} / \mathrm{ml}$, remaining for 7 days with exchanging half of the media at every 2 days. One day after photochemotherapy, the culture media was removed from the wells (Tesplate 96F-TPP, Zollstrasse, Trasadingen, Switzerland) and $100 \mu \mathrm{l}$ of methanol was added and the plate, and incubated for 20 minutes at room temperature $\left(25^{\circ} \mathrm{C}\right)$. Methanol was then removed from the wells, being added $50 \mu \mathrm{l}$ of crystal violet dye $(0.5 \%$ crystal violet, $20 \%$ methanol, $80 \% \mathrm{dH}_{2} \mathrm{O}$, Sigma-Aldrich Corporation, Milwaukee, WI) followed by incubation for 10 minutes at room temperature. After that period, the dye was removed and the wells washed twice with tap water, followed by TWO immersions of the plate in a beaker filled with tap water. To extract de dye from the cells, $100 \mu \mathrm{l}$ of Sorenson's buffer (SDS) was added in the wells and the plate was incubated in a rotate shaker for 30 minutes. An ELISA-SpectraMax 190 Microplate Reader ${ }^{\odot}$ (Sunnyvale, CA) plate reader was used for the absorbance measurement at wavelength of $540 \mathrm{~nm}$.

\section{Statistical Analysis}

The data was analyzed using the Minitab15 software (Minitab, Belo Horizonte, MG, Brazil). Significance level was fixed in $5 \%$.

\section{RESULTS}

From crystal violet toxicity assay, we observed that MB and TBO presented a very similar pattern of toxicity. When compared to control group, both have a trend to decrease the cells viability along with concentration increasing, although this effect was showed to be significant only at $20 \mu \mathrm{g} / \mathrm{ml}$. Thus, concentrations below these levels were chosen from both phenotiazines for PACT assays.

The overall analysis showed that, the use of energy density of $4.2 \mathrm{~J} / \mathrm{cm}^{2}$ achieved the best results of lethality when using incubation time of 5 minutes and using either $10 \mu \mathrm{g} / \mathrm{ml}$ of TBO or MB-TBO. When the incubation time was increased to 60 minutes, all compounds are similarly effective, independent of the energy density used. So the use of either $10 \mu \mathrm{g} / \mathrm{ml}$ of TBO or MB-TBO during 5 minutes prior irradiation with $4.2 \mathrm{~J} / \mathrm{cm}^{2}, \lambda=660 \mathrm{~nm}$ laser light was the most efficient protocol (Table 1).

\section{DISCUSSION}

According to the WHO, leishmaniasis is among the six most important tropical diseases, affecting around 12 million people in 88 countries [16]. Chemotherapy remains the most effective control measure for this disease. The therapeutic arsenal routinely employed to treat patients with leishmaniasis is limited and unsatisfactory. For cutaneous leishmaniasis, pentavalent antimonials (sodium stibogluconate or meglumine antimoniate) are the first line therapeutic scheme recommended by the WHO, the second drug of choice (amphotericin B) and the third drug of choice is pentamidine. All of these medications are parenterally administered and may result in mild to severe side effects. These compounds are highly toxic, poorly tolerated, require long-term treatment and their effectiveness highly variable [17-19].

Pentavalent antimonials may cause hyperamylasemia, ECG abnormalities, bone marrow suppression and hepatotoxicity, as well as constitutional symptoms, such as myalgia, arthralgia, headache, fever, nausea, vomiting, and pain at the site of drug application, when administered intramuscularly. Amphotericin B may result in anemia, cardiac and nephrotoxic effects, hypokalemia, and constitutional side effects, such as nausea, vomiting, phlebitis, shivering, and fever, which sometimes require the interruption of treatment. Pentamidine is known for its cardiac toxicity, nephrotoxicity, hypotension, hypoglycemia, but the major concern is the possibility of development of diabetes mellitus [19].

Antimicrobial chemotherapy (PACT) which may also be called photosensitization lethal laser (PLL), based on topical or systemic non-toxic dye sensitive to light irradiation followed by at low doses with visible light of appropriate wavelength [20]. The photodynamic inactivation can be performed in a localized way, different from photodynamic therapy against cancer, where the compound photosensitizer is usually injected into the bloodstream and accumulates in the tumor, photodynamic inactivation may be made by local employment in the area of the photosensitizer infected by topical application, instillation, or interstitial injection [21].

In the presence of oxygen found in the cells, photosensitizer (FS) enabled can react with molecules in their neighborhood by electron transfer or hydrogen, leading to

TABLE 1. Percentage Lethality for Each Parameter Tested (Photosensitizer, Photosensitizer Concentration, Incubation Time, and Energy Density).

\begin{tabular}{|c|c|c|c|c|}
\hline \multirow[b]{2}{*}{ Photosensitizer $(\mu \mathrm{g} / \mathrm{ml})$} & \multicolumn{4}{|c|}{ Incubation time (minutes)/energy density $\left(\mathrm{J} / \mathrm{cm}^{2}\right)$} \\
\hline & $5 / 4.2(\%)$ & $5 / 8.4(\%)$ & $60 / 4.2(\%)$ & $60 / 8.4(\%)$ \\
\hline $\mathrm{MB}(5)$ & 95.72 & 93.89 & 98.51 & 95.59 \\
\hline TBO (5) & 97.71 & 92.59 & 98.58 & 97.11 \\
\hline MB-TBO (5) & 97.00 & 93.89 & 98.34 & 96.67 \\
\hline $\mathrm{MB}(10)$ & 97.91 & 92.59 & 98.31 & 96.67 \\
\hline TBO (10) & 99.24 & 93.15 & 98.31 & 97.11 \\
\hline MB-TBO (10) & 98.50 & 93.33 & 98.38 & 98.33 \\
\hline
\end{tabular}


production of free radicals (type I reaction) or by energy transfer to oxygen, leading to production of singlet oxygen (type II reaction). Both paths may lead to cell death and the destruction of diseased tissue [22-24].

Reagent oxygen species (ROS) simultaneously attack multiple molecules of varied properties. PACT is considered to have the potential to circumvent drug-resistance that is common to both infectious and non-infectious diseases. PACT should not cause resistance to the drugs used and deserves special attention as a new strategy to circumvent this so common problem encountered in the chemotherapy of some diseases [25].

The light sources can be used to bring the excitement of photosensitizing molecules, such as pulsed light, lights incoherent, xenon lamp, among others, all with specific filters that let you select the wavelength with greater penetration in tissues and of maximum absorption of the drug [26,27]. The radiation used often is provided by a laser system, which are preferably employed because they have specific characteristics that differ from the light as being monochromatic, coherent and collimated [28].

$\mathrm{MB}$ and TBO are photosensitizing phenothiazine accepted at the practice medical and demonstrated in studies in literature. Exhibit intense absorption of 600 $660 \mathrm{~nm}$, region of the spectrum useful in PACT to be in the "therapeutic window" required for efficient penetration of light in tissues [29,30]. Although its properties physical and chemical are similar the efficiency photodynamic of compounds, varies between the several microorganisms [23]. The use of staining agents such as MB and TBO in the analysis of the pathology of the microbial diseased state is well established, as mentioned above [31].

The phenothiazine dye toluidine blue $\mathrm{O}$ (TBO) is a heterocyclic aromatic colorant soluble in water or alcohol, which absorbs strongly in the region of the ultravioletvisible. A photosensitizing agent that is very effective in inactivating pathogenic organisms. TBO is a phenothiazine analogous to $\mathrm{MB}$ with minimal structural differences. It has been widely used in biological staining, especially in hematology [31]. TBO is a phenothiazine dye that interacts with biological molecules and mediators. Previous studies have demonstrated the high ability of TBO molecules to bind to DNA. DNA is an important target in the phenothiazinium dye photosensitized biological damage, and guanine residues. Resonance light scattering spectra of TBO in the presence of DNA indicated that TBO could aggregate along DNA surface [23].

TBO in solution is playing a highly active role in the light-mediated killing of the cells. There are two possible explanations for this observation. First, it may be due to the light-mediated extracellular generation of ROS (particularly singlet oxygen) that may diffuse itself into the cell producing damage cell death. The second explanation is that the first part of the photodynamic inactivationPDI process produces some damage at the cell surface that allows more TBO molecules present in solution to bind and/or penetrate the cell and therefore increasing the potency of the PDI process. The finding that there was a significant dependence of killing on cell density also implies that there is binding or interaction of the cells with a limited concentration of some species and that this occurs whether or not the PS is washed out from the cell suspension [23].

The methylene blue (MB) has shown to be an effective broad-spectrum photoantimicrobial agent [32]. Due to the hydrophilic nature of the outer cell membrane, MB acts leading both to a lack of activity against intracellular targets as membrane photodamage, hemolysis, etc. MB is a long established biological dye and has been known as a photoantimicrobial since 1928. The antiprotozoal activity of MB includes both African and American trypanosomiasis and leishmaniasis. The continuing use of MB as a lead compound for antimalarial research underlines its activity against Plasmodium spp. [33].

The electronically excited MB molecule is relatively stable and can undergo an electronic rearrangement. The excited form is known as an excited triplet state and it is this that facilitates photodynamic action. A relatively long-lived excited triplet state allows the excited MB molecule to pass on the excitational energy to other molecules. Direct interaction with adjacent molecules leads to electron transfer or hydrogen abstraction and the formation of radical species. This is known as Type I photosensitization, and with membrane-related molecules leads to the formation of lipid hydroperoxides and thus to membrane damage [22]. If the excited triplet state molecule interacts directly with oxygen, the excitational energy is transferred causing the formation of a highly reactive species, singlet oxygen. Formed in situ within a cellular environment, singlet oxygen is highly toxic on account of its oxidizing activity, although it has a very short lifetime and will decay to the ground state (non-toxic triplet oxygen) within microseconds. Singlet oxygen-mediated photosensitization is known as the Type II pathway [34].

Phenothiazines possess great affinity by trypanosomatides [35-37]. Trypanothione and trypanothione reductase (TryR)-based redox metabolism found in Leishmania sp. and other trypanosomatides exemplify the unique features of this group of organisms. Its absence in mammalian hosts, together with the sensitivity of trypanosomes against oxidative stress, makes this enzyme a unique target for exploitation for potential antileishmanial chemotherapeutics [34,38-40]. Trypanothione reductase (TR) was irreversibly inhibited phenothiazine derivatives. The inactivation of TR depends on (a) of incubation with the phenothiazine derivatives (b) the free radicals formed (c) and the concentration and structure of phenothiazine derivatives [38].

Another enzyme found in Leishmania sp., is the superoxide dismutase (SOD) SOD has its mechanism of action involved in anti-oxidant enzymes which catalyze the dismutation of $\mathrm{O}_{2}^{-}$to $\mathrm{H}_{2} \mathrm{O}_{2}$ and $\mathrm{O}_{2}$ protecting catalase and peroxidase against inhibition by $\mathrm{O}_{2}{ }^{-}$, which can be derived from the phenothiazine [41,42].

Based on this knowledge about the actions of phenothiazine derivatives on structures and enzymes of Leishmania, we can better understand why the choice of some works of PACT with MB $[43,44]$. 
Other advantages of PACT are: the procedure can be repeated several times if necessary, since no toxic effects and is usually non-invasive. In addition, due to its low risk, can be used in people who are elderly or frail to undergo other surgery. The main side effect is sensitization to light, which is circumvented by avoiding exposure to sunlight and interior light too intense for a period of up to 6 weeks depending on the photosensitizer [45].

Because of its ability to interact at a more internal $\mathrm{MB}$, we decided to evaluate the TBO, individually and combined with $\mathrm{MB}$ for comparison with results previously available in the literature with MB. Our results showed that TBO really stands out when compared to MB. The contribution that this work can also provide for new research PACT with Leishmania, especially L. braziliensis, which is in respect to the parameters used in the irradiation for stimulation of the compounds used together with the low concentration of photosensitizers.

The concentrations used were well below those considered toxic based on toxicity test with crystal violet, giving us greater security to work and to modify the concentrations without causing problems cytotoxicity. Another point worth highlighting in this work is the total time for which the technique is carried out. We conducted four experimental periods, one where the child understands the pre-incubation of 5 minutes with 2 minutes of irradiation, a total of 7 minutes of dealing with a dose of $4.2 \mathrm{~J} / \mathrm{cm}^{2}$. Already the longest period includes a pre-incubation of 60 minutes with 4 minutes of irradiation, totaling 64 minutes of procedure and a dose of $8.4 \mathrm{~J} / \mathrm{cm}^{2}$. As there was no statistical difference of the best concentration, $10 \mu \mathrm{g} / \mathrm{ml}$ of TBO compared the times of pre-incubation of 5 or 60 minutes, we suggest that among these possibilities tested at work, the best procedure would be to use $10 \mu \mathrm{g} /$ $\mathrm{ml}$ of TBO irradiated by a laser device with a dose of $4.2 \mathrm{~J} / \mathrm{cm}^{2}$. In this procedure, we have less time with greater lethality.

\section{REFERENCES}

1. De Muylder G, Ang KKH, Chen S, Arkin MR, Engel JC, McKerrow JH. A screen against Leishmania intracellular amastigotes: Comparison to a promastigote screen and identification of a host cell-specific hit. PLoS Negl Trop Dis 2011;5:e1253.

2. do Monte-Neto RL, Coelho AC, Raymond F, Légaré D, Corbeil J, Melo MN, Frézard F, Ouellette M. Gene expression profiling and molecular characterization of antimony resistance in Leishmania amazonensis. PLoS Negl Trop Dis 2011;5:e1167.

3. Santos DO, Coutinho MFM, Bottino CG, Vieira RT, Nascimento SB, Bernardino A, Bourguignon SC, Corte-Real S, Pinho RT, Rodrigues CR, Castro HC. Leishmaniasis treatment-A challenge that remains: A review. Parasitol Res 2008;103:1-10.

4. Machado PR, Ampuero J, Guimarães LH, Villasboas L, Rocha AT, Schriefer A, Sousa RS, Talhari A, Penna G, Carvalho EM. Miltefosine in the treatment of cutaneous leishmaniasis caused by Leishmania braziliensis in Brazil: A randomized and controlled trial. PLoS Negl Trop Dis 2010;4(12):e912.

5. Tavares NM, Silva RA, Costa DJ, Pitombo MA, Fukutani KF, Miranda JC, Valenzuela JG, Barral A, de Oliveira CI, Barral-Netto M, Brodskyn C. Lutzomyia longipalpis saliva or salivary protein LJM19 Protects against Leishmania braziliensis and the saliva of its vector, lutzomyia intermedia. PLoS Negl Trop Dis 2011;5(5):e1169.

6. Soto J, Soto P. Current situation and future of anti-leishmanial therapy in Colombia. Biomedica 2006;26:194-206.

7. Machado-Pinto J, Pinto J, da Costa CA, Genaro O, Marques MJ, Modabber F, Mayrink W. Immunochemotherapy for cutaneous leishmaniasis: A controlled trial using killed Leishmania (Leishmania) amazonensis vaccine plus antimonial. Int J Dermatol 2002;41:73-78.

8. Vélez I, López L, Sánchez X, Mestra L, Rojas C, Rodríguez E. Efficacy of miltefosine for the treatment of American cutaneous Leishmaniasis. Am J Trop Med Hyg 2010;83:351-356.

9. Enk CD, Fritsch C, Jonas F, Nasereddin A, Ingber A, Jaffe CL. Ruzicka T. Treatment of cutaneous leishmaniasis with photodynamic therapy. Arch Dermatol 2003;139:432-434.

10. Gardlo K, Horska Z, Enk CD, Rauch L, Megahed M, Ruzicka T, Fritsch C. Treatment of cutaneous leishmaniasis by photodynamic therapy. J Am Acad Dermatol 2003;48:893896.

11. Dutta S, Ray D, Kolli BK, Chang KP. Photodynamic sensitization of Leishmania amazonensis in both extracellular and intracellular stages with aluminum phthalocyanine chloride for photolysis in vitro. Antimicrob Agents Chemotherap 2005;49:4474-4484.

12. Montanari J, Maidana C, Esteva MI, Salomon C, Morilla MJ, Romero EL. Sunlight triggered photodynamic ultradeformable liposomes against Leishmania braziliensis are also leishmanicidal in the dark. J. Control Release 2010;147:368376.

13. Nitzan Y, Wexler HM, Finegold SM. Inactivation of anaerobic bacteria by various photosensitized porphyrins or by hemin. Curr Microbiol 1994;29:125-131.

14. Calzavara-Pinton PG, Venturini M, Sala R. A comprehensive overview of photodynamic therapy in the treatment of superficial fungal infections of the skin. J Photochem Photobiol B 2005;78:1-6.

15. Pluta K, Mlodawska BM, Jelen M. Recent progress in biological activities of synthesized phenothiazines. Eur J Med Chem 2011;46:3179-3189.

16. Bailey MS, Lockwood DN. Cutaneous leishmaniasis. Clin Dermatol 2007;25:203-211.

17. Foroumadi A, Pournourmohammadi S, Soltani F, Asgharian-Rezaee M, Dabiri S, Kharazmi A, Shafiee A. Synthesis and in vitro leishmanicidal activity of 2-(5-nitro-2-furyl) and 2-(5-nitro-2-thienyl)-5-substituted-1,3,4-thiadiazoles. Bioorg Med Chem Lett 2005;15:1983-1985.

18. Zauli-Nascimento RC, Miguel DC, Yokoyama-Yasunaka JKU, Pereira LIA, Oliveira MAP, Ribeiro-Dias F, Dorta ML, Uliana SR. In vitro sensitivity of Leishmania (Viannia) braziliensis and Leishmania (Leishmania) amazonensis Brazilian isolates to meglumine antimoniate and amphotericin B. Trop Med Int Health 2010;15:68-76.

19. Pimentel MIF, Baptista C, Rubin EF, Vasconcellos ECF, Lyra MR, Salgueiro Mde M, Saheki MN, Rosalino CM, Madeira de F, Silva AF, Confort EM, Schubach Ade O. American cutaneous leishmaniasis caused by Leishmania (Viannia) braziliensis resistant to meglumine antimoniate, but with good response to pentamidine: A case report. Rev Soc Bras Med Trop 2011;44:254-256.

20. Gad F, Zahra T, Hasan T, Hamblin MR. Photodynamic inactivation of Gram-positive pathogenic bacteria: Effect of growth phase and extracellular slime. Antimicrob Agents Chemother 2004;48:2173-2178.

21. Hamblin MR, Hasan T. Photodynamic therapy: A new antimicrobial approach to infectious disease? Photochem Photobiol Sci 2004;3:436-450.

22. Barbosa AFS, Soares LGP, Aciole JMS, Aciole GTS, Pitta IR, Galdino SL, Pinheiro ALB. Evaluation of photodynamic antimicrobial therapy (PACT) against trypomastigotes of Trypanosoma cruzi: In vitro study. AIP Conf Proc 2011;1364:5559 .

23. Demidova TN, Hamblin MR. Effect of cell-photosensitizer binding and cell density on microbial photoinactivation. Antimicrob Agents Chemother 2005;49:2329-2335.

24. Lambrechts SAG, Aalders MCG, Marle JV. Mechanistic study of the photodynamic inactivation of Candida albicans 
by a cationic porphyrin. Antimicrob Agents Chemother 2005;49:2026-2034.

25. Dutta S, Ongarora BG, Li H, Vicente MGH, Kolli BK, Chang KP. Intracellular targeting specificity of novel phthalocyanines assessed in a host parasite model for developing potential photodynamic medicine. PLoS ONE 2011;6:e20786.

26. Machado AEH. Photodynamic therapy principles, potential of application and perspectives. Quím Nova 2000;23:237243.

27. Mang TS. Lasers and light sources for PDT: Past, present and future. Photodiag Photodyn Therapy 2004;1:43-48

28. Pierce LA. Laser physics physiology. Plast Surg Nurs 1997;17:123-128.

29. Wainwright M, Grice NJ, Pye LEC. Phenothiazine photosensitisers. Part II. 3 7-bis(arylamino)phenothiazines. Dyes Pigments 1999;42:45-51.

30. Sommer AP, Pinheiro AL, Mester AR, Franke RP, Whelan HT. Biostimulatory windows in low-intensity laser activation: Lasers, scanners, and NASA's light-emitting diode array system. J Clin Med Laser Surg 2001;19:29-33.

31. Wainwright $M$. The development of phenothiazinium photosensitisers. Photodiagnosis Photodyn Ther 2005;2:263-272.

32. Wainwright M, Mohr H, Walker W. Phenothiazinium derivatives for pathogen inactivation in blood products. J Photochem Photobiol B 2007;86:45-58.

33. Wainwright M, Baptista MS. The application of photosensitisers to tropical pathogens in the blood supply. Photodiagnosis Photodyn Ther 2011;8:240-248.

34. Ilanchelian M, Ramaraj R. Binding interactions of toluidine blue $\mathrm{O}$ with Escherichia coli DNA: Formation of bridged structure. J Fluoresc 2011;21:1439-1453.

35. T'ung T. In vitro photodynamic action of methylene blue on Trypanosoma brucei. Proc Soc Exp Biol Med 1938;38:29-31.

36. Ferreira CS, Bezerra RC, Pinheiro AA. Methylene blue vital staining for Trypanosoma cruzi trypomastigotes and epimastigotes. Rev Inst Med Trop 2006;48:347-349.

37. Buchholz K, Comini MA, Wissenbach D, Schirmer RH, KrauthSiegel RL, Gromer S. Cytotoxic interactions of methylene blue with trypanosomatid-specific disulfide reductases and their dithiol products. Mol Biochem Parasitol 2008;160:65-69.

38. Correa JG, Fairlamb AH, Stoppani AOM. Trypanosoma cruzi trypanothione reductase is inactivated by peroxidasegenerate phenothiazine cationic radicals. Free Rad Res 2001;34:363-368.

39. Parveen S, Kahn MOF, Austin SE, Croft SL, Yardley V, Rock P, Douglas KT. Antitrypanosomal, antileishmanial, and antimalarial activities of quaternary arylalkylammonium 2-amino-4-chlorophenyl phenyl sulfides, a new class of trypanothione reductase inhibitor, and of $N$-acyl derivatives of 2-amino-4-chlorophenyl phenyl sulfide. J Med Chem 2005; 48:8087-8097.

40. Lima LM, Barreiro EJ. Bioisosterism: A useful strategy for molecular modification and drug design. Curr Med Chem $2005 ; 12: 23-49$.

41. Kim SY, Kwon OJ, Park JW. Inactivation of catalase and superoxide dismutase by singlet oxygen derived from photoactivated dye. Biochemie 2001;83:437-444.

42. Khouri R, Bafica A, Silva Mda P, Noronha A, Kolb JP, Wietzerbin J, Barral A, Barral-Netto M, Van Weyenbergh J. IFN- $\beta$ Impairs superoxide-dependent parasite killing in human macrophages: Evidence for a deleterious role of SOD1 in cutaneous leishmaniasis. J Immunol 2009;182: 2525-2531.

43. Peloi LS, Biondo CE, Kimura E, Politi MJ, Lonardoni MV, Aristides SM, Dorea RC, Hioka N, Silveira TG. Photodynam ic therapy for American cutaneous leishmaniasis: The efficacy of methylene blue in hamsters experimentally infected with Leishmania (Leishmania) amazonensis. Exp Parasitol 2011;128:353-356.

44. Song D, Lindoso JA, Oyafuso LK, Kanashiro EH, Cardoso JL, Uchoa AF, Tardivo JP, Baptista MS. Photodynamic therapy using methylene blueto treat cutaneous leishmaniasis. Photomed Laser Surg 2011;29:711-715.

45. Capella MA Capella L. A light in multidrug resistance: Photodynamic treatment of multidrug-resistant tumors. J Biomed Sci 2003;10:361-366. 